\title{
Construction of compact-integral operators on $B C(\Omega)$ with application to the solvability of functional integral equations
}

\author{
Reza Allahyari Reza Arab* Ali Shole Haghighi
}

\begin{abstract}
In this article, using the concept of measure of noncompactness, we give some results concerning the compactness and continuity of the nonlinear Volterra and Fredholm integral operators on the space $B C(\Omega)(\Omega$ is an unbounded subset of the Euclidean space $\mathbb{R}^{n}$ ). Then, we prove an existence result for a functional integral equation which includes several classes of nonlinear integral equations. Our results generalize and improve some previous works. We will also include some examples which show that our results are applicable where the previous ones are not.
\end{abstract}

\section{Introduction}

Fixed point theory has two main branches: metric fixed point theory and topological fixed point theory. Concerning the topological branch, the concept of the compactness plays a fundamental role in creating the basis of several investigations conducted in nonlinear analysis and is very useful in several topics of applied mathematics, engineering, mathematical physics, numerical analysis, and so on.

On the other hand, the measures of noncompactness are very useful tools in functional analysis, for instance, in the theory of operator equations in Banach spaces.

\footnotetext{
*Corresponding author

Received by the editors in April 2015 - In revised form in June 2015.

Communicated by G. Godefroy.

2010 Mathematics Subject Classification : 47H09, 47H10, 34A12.

Key words and phrases : Measure of noncompactness, Functional integral equations, Fixed point, Compact-integral operators.
} 
They are also used in the study of functional equations, ordinary and partial differential equations, fractional partial differential equations, integral and integrodifferential equations, optimal control theory, etc. In particular, the fixed point theorems derived from them have many applications. There exists a considerable literature devoted to this subject (see for example [1-7, 10, 11, 13-15, 17-20]). Integral equations play a very important role in nonlinear analysis and find numerous applications in engineering, mathematical physics, economics, etc. Recently, in [4] Aghajani and Jalilian extended results of the papers [11, 14] by considering the following integral equation in general form

$$
x(t)=f\left(t, x(\alpha(t)), \int_{0}^{\beta(t)} g(t, s, x(\gamma(s))) d s\right)
$$

on $B C\left(\mathbb{R}_{+}\right)$.

Moreover, Aghajani et al. [7] and Arab [8] studied the existence of solutions for the functional integral equations on $B C\left(\mathbb{R}_{+} \times \mathbb{R}_{+}\right)$

$$
\begin{aligned}
x(t, s) & =f\left(t, s, x\left(\xi_{1}(t), \xi_{2}(s)\right), \int_{0}^{\beta_{2}(s)} \int_{0}^{\beta_{1}(t)} g_{1}\left(t, s, v, w, x\left(\eta_{1}(v), \eta_{2}(w)\right)\right) d v d w,\right. \\
& \left.\int_{0}^{\beta_{3}(t)} g_{2}\left(t, s, v, x\left(\zeta_{1}(v), \zeta_{2}(s)\right)\right) d v\right)
\end{aligned}
$$

and

$$
\begin{aligned}
x(t, s) & =a(t, s)+h_{1}(t, s, x(t, s)) \int_{0}^{t} g_{1}(t, s, u) f_{1}(u, x(u, s)) d u \\
& +h_{2}(t, s, x(t, s)) \int_{0}^{t} \int_{0}^{s} g_{2}(t, s, u, v) f_{2}(u, v, x(u, v)) d u d v
\end{aligned}
$$

respectively.

Also, equations like

$$
x(t)=f\left(t, \int_{0}^{t} u(t, s, x(s)) d s, \int_{0}^{\infty} a_{2}(t) v(s, x(s)) d s\right), t \geq 0
$$

and

$$
x(t)=p(t)+f(t, x(t)) \int_{0}^{\infty} g(t, \tau) h(\tau, x(\tau)) d \tau, \quad t \geq 0
$$

have been considered by Aghajani et al. [5] and Banaś et al. [16], respectively. In this paper, we study the existence of solutions for the following functional integral equations

$$
u(x)=f\left(x, u(x), \int_{\Gamma(x)} g(x, y, u(\xi(y))) d y, \int_{\Omega^{\prime}} k(x, y) h(y, u(\eta(y))) d y\right),
$$

and

$$
u(x)=f_{1}(x, u(x))+f_{2}(x, u(x)) \int_{\Omega^{\prime}} \int_{\Gamma(x)} k(x, y) g(y, z, u(\zeta(y, z))) d z d y .
$$

We are going to show that those equations have solutions belonging to the space $B C(\Omega)$ where $x \in \Omega\left(\Omega\right.$ is an unbounded subset of the Euclidean space $\left.\mathbb{R}^{n}\right)$. 
Those equations when $\Omega=\mathbb{R}_{+}$and $\Omega=\mathbb{R}_{+} \times \mathbb{R}_{+}$respectively reduce to the functional integral equations on $B C\left(\mathbb{R}_{+}\right)$and functional integral equations on $B C\left(\mathbb{R}_{+} \times \mathbb{R}_{+}\right)$.

Let us notice that this equations are general forms of many above-mentioned integral equations, for example, Eq. (1.7) is a general form of Eq. (1.5) (consider $u(x)=x(t), f_{1}(x, u(x))=p(t), f_{2}(x, u(x))=f(t, x(t)), \Omega^{\prime}=[0, \infty)$, $\Gamma(x)=[0,1], k(x, y)=g(t, \tau)$ and $g(y, z, u(\zeta(y, z)))=h(\tau, x(\tau)))$, and in a similar method, Eq. (1.6) is a general form of integral equations Eq. (1.1), Eq. (1.2) and Eq. (1.4).

Now, for investigating the existence of solutions for this equations, in Section 2 we present some definitions and preliminary results about the concept of measure of noncompactness, in Section 3 we obtain main results about the compactness and continuity of Volterra and Fredholm integral operators, and finally in Section 4 we prove an existence result for this equations. The results of this paper generalize and improve several main results ( see $[1,3,4,6,7,11,14,17,18,20]$ ). We will also include some examples which show that our results are applicable where the previous ones are not.

\section{Preliminaries}

Here, we recall some basic facts concerning measures of noncompactness from [12], which is defined axiomatically in terms of some natural conditions. Denote by $\mathbb{R}$ the set of real numbers and put $\mathbb{R}_{+}=[0,+\infty)$. Let $(E,\|\cdot\|)$ be a real Banach space with zero element 0 . Let $\bar{B}(x, r)$ denote the closed ball centered at $x$ with radius $r$. The symbol $\bar{B}_{r}$ stands for the ball $\bar{B}(0, r)$. For $X$, a nonempty subset of $E$, we denote by $\bar{X}$ and Conv $X$ the closure and the closed convex hull of $X$, respectively. Moreover, let us denote by $\mathfrak{M}_{E}$ the family of nonempty bounded subsets of $E$ and by $\mathfrak{N}_{E}$ its subfamily consisting of all relatively compact subsets of $E$.

Definition 2.1. A mapping $\mu: \mathfrak{M}_{E} \longrightarrow \mathbb{R}_{+}$is said to be a measure of noncompactness in $E$ if it satisfies the following conditions:

$1^{\circ}$ The family $\operatorname{ker} \mu=\left\{X \in \mathfrak{M}_{E}: \mu(X)=0\right\}$ is nonempty and $\operatorname{ker} \mu \subseteq \mathfrak{N}_{E}$.

$2^{\circ} X \subset Y \Longrightarrow \mu(X) \leq \mu(Y)$.

$3^{\circ} \mu(\bar{X})=\mu(X)$.

$4^{\circ} \mu(\operatorname{Conv} X)=\mu(X)$.

$5^{\circ} \mu(\lambda X+(1-\lambda) Y) \leq \lambda \mu(X)+(1-\lambda) \mu(Y)$ for $\lambda \in[0,1]$.

$6^{\circ}$ If $\left\{X_{n}\right\}$ is a sequence of closed sets from $\mathfrak{M}_{E}$ such that $X_{n+1} \subset X_{n}$ for $n=1,2, \cdots$ and if $\lim _{n \rightarrow \infty} \mu\left(X_{n}\right)=0$ then $X_{\infty}=\cap_{n=1}^{\infty} X_{n} \neq \varnothing$.

Definition 2.2. Let $X$ be a Banach space. An operator (not necessarily linear) $F: X \longrightarrow$ $X$ is compact if the closure of $F(Y)$ is compact whenever $Y \subset X$ is bounded. 
Remark 2.1. Let $C$ be any nonempty and bounded subsets of a Banach space $E$ and let $T: E \longrightarrow E$ be a continuous mapping, if $\mu(T(C))=0$, where $\mu$ is an arbitrary measure of noncompactness, then by using Definition $2.1, T: E \longrightarrow E$ is a compact operator.

Now we recall the following important fixed point theorem playing a key role in the sequel.

Theorem 2.1. [3] Let $C$ be a nonempty, bounded, closed and convex subset of a Banach space $E$ and let $F: C \longrightarrow C$ be a continuous mapping such that

$$
\mu(F X) \leq \varphi(\mu(X))
$$

for any nonempty subset $X$ of $C$ where $\mu$ is an arbitrary measure of noncompactness and $\varphi: \mathbb{R}_{+} \longrightarrow \mathbb{R}_{+}$is a nondecreasing function such that $\varphi(t)<t$ for each $t \geq 0$ and $\varphi(0)=0$. Then $F$ has at least one fixed point in the set $C$.

The set $\Omega$ is a nonempty and unbounded subset of the Euclidean space $\mathbb{R}^{n}$. Let $B C(\Omega)$ be the Banach space of all bounded and continuous functions on $\Omega$ equipped with the standard norm

$$
\|u\|_{u}=\sup \{|u(x)|: x \in \Omega\} .
$$

For any nonempty bounded subset $U$ of $B C(\Omega), u \in U, T>0$ and $\varepsilon \geq 0$

$$
\begin{aligned}
& \omega^{T}(u, \varepsilon)=\sup \left\{|u(x)-u(y)|: x, y \in \bar{B}_{T},\|x-y\| \leq \varepsilon\right\} . \\
& \omega^{T}(U, \varepsilon)=\sup \left\{\omega^{T}(u, \varepsilon): u \in U\right\}, \\
& \omega_{0}^{T}(U)=\lim _{\varepsilon \rightarrow 0} \omega^{T}(U, \varepsilon), \omega_{0}(U)=\lim _{T \rightarrow \infty} \omega_{0}^{T}(U), \\
& U(x)=\{u(x): u \in U\},
\end{aligned}
$$

and

$$
\mu(U)=\omega_{0}(U)+\limsup _{\|x\| \rightarrow \infty} \operatorname{diam} U(x) .
$$

Similar to [9] (cf. also [12]), it can be shown that the function $\mu$ is a measure of noncompactness in the space $B C(\Omega)$ (in the sense of Definition 2.1).

\section{Construction of compact-integral operator on $B C(\Omega)$}

In this section we obtain main results about the compactness and continuity of Volterra and Fredholm integral operators.

Definition 3.1. Let $\Omega$ be an unbounded subset of the Euclidean space $\mathbb{R}^{n}$. We say that a mapping $\Lambda: \Omega \longrightarrow \mathfrak{M}_{\mathbb{R}^{m}}$ is continuous function if for each $\varepsilon>0$ there exists $\delta>0$ such that

$$
\|x-y\|<\delta \Longrightarrow m(\Lambda(x) \triangle \Lambda(y))<\varepsilon
$$

where $m$ is Lebesgue measure on $\Omega$ and $\triangle$ denotes the symmetric difference. 
First, we define a compact-integral operator of Volterra type on $B C(\Omega)$.

Theorem 3.1. Assume that the following conditions are satisfied:

(i) Let $\Omega$ be a nonempty and unbounded subset of the Euclidean space $\mathbb{R}^{n}, \Gamma: \Omega \longrightarrow$ $\mathfrak{M}_{\mathbb{R}^{m}}$ and $\xi: \bigcup_{x \in \Omega} \Gamma(x) \longrightarrow \Omega$ are continuous functions such that $\bigcup_{\|x\| \leq T} \Gamma(x)$ is bounded subset of $\mathbb{R}^{m}$ for all $T>0$.

(ii) $g: \Omega \times\left(\bigcup_{x \in \Omega} \Gamma(x)\right) \times \mathbb{R} \longrightarrow \mathbb{R}$ is continuous and there exists a nondecreasing and continuous function $\vartheta: \mathbb{R}_{+} \longrightarrow \mathbb{R}_{+}$such that

$$
\sup \left\{\left|\int_{\Gamma(x)} g(x, y, u(\xi(y))) d y\right|: x \in \Omega, u \in \bar{B}_{r}\right\} \leq \vartheta(r)
$$

Moreover, for any $r \in \mathbb{R}_{+}$

$$
\lim _{\|x\| \rightarrow \infty}\left|\int_{\Gamma(x)}[g(x, y, u(\xi(y)))-g(x, y, v(\xi(y)))] d y\right|=0
$$

uniformly with respect to $u, v \in \bar{B}_{r}$.

Then $G: B C(\Omega) \longrightarrow B C(\Omega)$ defined by

$$
G u(x)=\int_{\Gamma(x)} g(x, y, u(\xi(y))) d y
$$

is a compact and continuous operator and $\|G u\|_{u} \leq \vartheta\left(\|u\|_{u}\right)$.

Proof. Obviously, $G u(x)$ for any $u \in B C(\Omega)$ is continuous on $\Omega$ and by (3.1), $G$ is a self operator on $B C(\Omega)$. Now we show that $G$ is continuous. To verify this, take $u \in B C(\Omega)$ and $\varepsilon>0$ arbitrarily. Moreover take $v \in B C(\Omega)$ with $\|u-v\|_{u}<\varepsilon$. Then, considering condition (ii) there exists $T>0$ such that for any $x \in \Omega$ such that $\|x\|>T$ we have

$$
|G u(x)-G v(x)| \leq \mid \int_{\Gamma(x)}[g(x, y, u(\xi(y))-g(x, y, u, v(\xi(y))] d y \mid \leq \varepsilon .
$$

Also, if $x \in \bar{B}_{T}$, then we obtain

$$
|G u(x)-G v(x)| \leq\left|\int_{\Gamma(x)}\right| g\left(x, y, u(\xi(y))-g\left(x, y, v(\xi(y))|d y| \leq \Gamma_{T} \theta_{T}(\varepsilon),\right.\right.
$$

where

$$
\begin{aligned}
& \Gamma_{T}=\sup \left\{m(\Gamma(x)): x \in \bar{B}_{T}\right\} \\
& \theta_{T}(\varepsilon)=\sup \left\{|g(x, y, u)-g(x, y, v)|: x \in \bar{B}_{T}, y \in \overline{\bigcup_{\|x\| \leq T} \Gamma(x)}, u, v \in[-b, b],\right. \\
& \left.\|u-v\|_{u} \leq \varepsilon\right\}
\end{aligned}
$$


with $b=\|u\|_{u}+\varepsilon$. By using the continuity of $g$ on the compact set $\bar{B}_{T} \times \overline{\bigcup_{\|x\| \leq T} \Gamma(x)} \times[-b, b]$, we have $\theta_{T}(\varepsilon) \longrightarrow 0$ as $\varepsilon \longrightarrow 0$. Thus, $G$ is a continuous function on $B C(\Omega)$. To complete the proof we need to verify that $G$ is a compact operator. Let $U$ be a nonempty and bounded subset of $B C(\Omega)$, and assume that $T>0$ and $\varepsilon>0$ are arbitrary constants. Then for $u \in U$ and $x_{1}, x_{2} \in \bar{B}_{T}$, with $\left\|x_{2}-x_{1}\right\| \leq \varepsilon$ we have

$$
\begin{aligned}
\left|G u\left(x_{2}\right)-G u\left(x_{1}\right)\right| \leq & \left|\int_{\Gamma\left(x_{1}\right)} g\left(x_{1}, y, u(\xi(y))\right) d y-\int_{\Gamma\left(x_{2}\right)} g\left(x_{1}, y, u(\xi(y))\right) d y\right| \\
& +\left|\int_{\Gamma\left(x_{2}\right)} g\left(x_{1}, y, u(\xi(y))\right) d y-\int_{\Gamma\left(x_{2}\right)} g\left(x_{2}, y, u(\xi(y))\right) d y\right| \\
\leq & \left|\int_{\Gamma\left(x_{1}\right) \triangle \Gamma\left(x_{2}\right)} g\left(x_{1}, y, u(\xi(y))\right) d y\right| \\
& +\int_{\Gamma\left(x_{2}\right)}\left|g\left(x_{1}, y, u(\xi(y))\right)-g\left(x_{2}, y, u(\xi(y))\right)\right| d y \\
\leq & U_{r}^{T} \omega^{T}(\Gamma, \varepsilon)+\Gamma_{T} \omega_{r}^{T}(g, \varepsilon),
\end{aligned}
$$

where

$$
\begin{aligned}
& r=\sup \left\{\|u\|_{u}: u \in U\right\}, \\
& \omega_{r}^{T}(g, \varepsilon)=\sup \left\{\left|g\left(x_{1}, y, u\right)-g\left(x_{2}, y, u\right)\right|: x_{1}, x_{2} \in \bar{B}_{T},\left\|x_{2}-x_{1}\right\| \leq \varepsilon,\right. \\
& \left.y \in \bigcup_{\|x\| \leq T} \Gamma(x), u \in[-r, r]\right\} \\
& \omega^{T}(\Gamma, \varepsilon)=\sup \left\{m(\Gamma(x) \triangle \Gamma(y)): x, y \in \bar{B}_{T},\|x-y\| \leq \varepsilon\right\} \\
& U_{r}^{T}=\sup \left\{|g(x, y, u)|: x \in \bar{B}_{T}, y \in \bigcup_{\|x\| \leq T} \Gamma(x), u \in[-r, r]\right\} .
\end{aligned}
$$

Since $u$ was an arbitrary element of $U$ in (3.3), we obtain

$$
\omega^{T}(G(U), \varepsilon) \leq \Gamma_{T} \omega_{r}^{T}(g, \varepsilon)+U_{r}^{T} \omega^{T}(\Gamma, \varepsilon)
$$

On the other hand, by using the uniform continuity of $g$ and $\Gamma$ on the compact sets $\bar{B}_{T} \times \overline{\bigcup_{\|x\| \leq T} \Gamma(x)} \times[-r, r]$ and $\bar{B}_{T}$, respectively, we have $\omega_{r}{ }^{T}(g, \varepsilon) \longrightarrow 0$ and $\omega^{T}(\Gamma, \varepsilon) \longrightarrow 0$ as $\varepsilon \longrightarrow 0$. Therefore, we obtain $\omega_{0}^{T}(G(U))=0$, which gives

$$
\omega_{0}(G(U))=0 .
$$

Moreover, for arbitrary $u, v \in U \subset \bar{B}_{r}$ and $x \in \Omega$ we have the following estimate

$$
|G u(x)-G v(x)| \leq\left|\int_{\Gamma(x)}[g(x, y, u(\xi(y)))-g(x, y, v(\xi(y)))] d y\right| .
$$

Thus, we obtain

$\operatorname{diam} G(U)(x) \leq \sup \left\{\left|\int_{\Gamma(x)}[g(x, y, u(\xi(y)))-g(x, y, v(\xi(y)))] d y\right|: u, v \in \bar{B}_{r}\right\}$

Taking $\|x\| \longrightarrow \infty$ in the inequality (3.6), then using (ii) we arrive at

$$
\limsup _{\|x\| \longrightarrow \infty} \operatorname{diam} G(U)(x)=0 .
$$


Further, combining (3.5) and (3.7) we get

$$
\limsup _{\|x\| \rightarrow \infty} \operatorname{diam} G(U)(x)+\omega_{0}(G(U))=0
$$

or, equivalently $\mu(G(U))=0$. Thus, $G$ is a compact operator. Also, by condition (ii) we deduce $\|G u\|_{u} \leq \vartheta\left(\|u\|_{u}\right)$ and the proof is complete.

Now, we define a compact-integral operator of Fredholm type on $B C(\Omega)$.

Theorem 3.2. Assume that the following conditions are satisfied:

(i) Let $\Omega$ be a nonempty and unbounded subset of the Euclidean space $\mathbb{R}^{n}$, $k: \Omega \times \Omega^{\prime} \longrightarrow \mathbb{R}\left(\Omega^{\prime}\right.$ is a subset of $\left.\mathbb{R}^{k}\right)$ and $\eta: \Omega^{\prime} \longrightarrow \Omega$ are continuous functions,

(ii) $h: \Omega^{\prime} \times \mathbb{R} \longrightarrow \mathbb{R}$ is continuous and there exist a continuous function $a: \Omega^{\prime} \longrightarrow$ $\mathbb{R}_{+}$and a continuous and nondecreasing function $b: \mathbb{R}_{+} \longrightarrow \mathbb{R}_{+}$such that

$$
|h(x, u)| \leq a(x) b(|u|)
$$

for $x \in \Omega^{\prime}$ and $u \in \mathbb{R}$. Also the function $y \longrightarrow a(y) k(x, y)$ is integrable over $\Omega^{\prime}$ for any fixed $x \in \Omega$,

(iii) There exists a positive constant D such that

$$
\sup \left\{\int_{\Omega^{\prime}} a(y)|k(x, y)| d y: x \in \Omega\right\}<D
$$

and

$$
\lim _{\|x\| \rightarrow \infty} \int_{\Omega^{\prime}} a(y)|k(x, y)| d y=0,
$$

(iv) The following equality holds:

$$
\lim _{T \rightarrow \infty}\left\{\sup \left\{\int_{\Omega^{\prime} \backslash \bar{B}_{T}} a(y)|k(x, y)| d y: x \in \Omega\right\}\right\}=0 .
$$

Then $H: B C(\Omega) \longrightarrow B C(\Omega)$ defined by

$$
H u(x)=\int_{\Omega^{\prime}} k(x, y) h(y, u(\eta(y))) d y
$$

is a compact and continuous operator and $\|H u\|_{u} \leq D b\left(\|u\|_{u}\right)$.

Proof. In view of the imposed assumptions we have that the function $H u(x)$ is continuous on $\Omega$ for any $u \in B C(\Omega)$, using our assumptions, we obtain

$$
\begin{aligned}
|H u(x)| & =\left|\int_{\Omega^{\prime}} k(x, y) h(y, u(\eta(y))) d y\right| \\
& \leq\left|\int_{\Omega^{\prime}} k(x, y) a(y) b(|u(\eta(y))|) d y\right| \\
& \leq b\left(\|u\|_{u}\right)\left|\int_{\Omega^{\prime}} k(x, y) a(y) d y\right| \\
& \leq D b\left(\|u\|_{u}\right)
\end{aligned}
$$


Hence by (iii), we have

$$
\|H u\|_{u} \leq D b\left(\|u\|_{u}\right)
$$

and $H$ is a self operator on $B C(\Omega)$. Now we show that $H$ is continuous. To verify this, take $u \in B C(\Omega)$ and $\varepsilon>0$ arbitrarily. Moreover take $v \in B C(\Omega)$ with $\|u-v\|_{u}<\varepsilon$. Then we have

$$
|H u(x)-H v(x)| \leq\left|\int_{\Omega^{\prime}} k(x, y)[h(y, u(\eta(y)))-h(y, v(\eta(y)))] d y\right| .
$$

This result together condition (iii) imply that there exists $T>0$ such that for all $x \in \Omega$ with $\|x\|>T$ we have

$$
\begin{aligned}
|H u(x)-H v(x)| & \leq \int_{\Omega^{\prime}} k(x, y)[|h(y, u(\eta(y)))|+|h(y, v(\eta(y)))|] d y \\
& \leq \int_{\Omega^{\prime}} 2 k(x, y) a(y) b\left(\|u\|_{u}+\varepsilon\right) d y \\
& \leq 2 D b\left(\|u\|_{u}+\varepsilon\right) \varepsilon .
\end{aligned}
$$

If $x \in \bar{B}_{T}$, then the inequality in (3.9) follows that

$$
\begin{aligned}
|H u(x)-H v(x)| \leq & \left|\int_{\bar{B}_{T}} k(x, y)[h(y, u(\eta(y)))-h(y, v(\eta(y)))] d y\right| \\
& +\left|\int_{\Omega^{\prime} \backslash \bar{B}_{T}} k(x, y)[h(y, u(\eta(y)))-h(y, v(\eta(y)))] d y\right| \\
\leq & m\left(\bar{B}_{T}\right) K_{T} \omega_{r_{0}}^{T}(h, \varepsilon)+2 b\left(r_{0}\right) \int_{\Omega^{\prime} \backslash \bar{B}_{T}}|k(x, y)||a(y)| d y
\end{aligned}
$$

where

$$
\begin{aligned}
& K_{T}=\sup \left\{k(x, y): x \in \bar{B}_{T} \subset \Omega, y \in \bar{B}_{T} \subset \Omega^{\prime}\right\} \\
& \omega_{r_{0}}^{T}(h, \varepsilon)=\sup \left\{|h(y, u)-h(y, v)|: y \in \bar{B}_{T} \subset \Omega^{\prime}, u, v \in\left[-r_{0}, r_{0}\right],\|u-v\|_{u} \leq \varepsilon\right\} .
\end{aligned}
$$

with $r_{0}=\|x\|+\varepsilon$. By using the continuity of $h$ on the compact set $\bar{B}_{T} \times\left[-r_{0}, r_{0}\right]$, we have $\omega_{r_{0}}^{T}(h, \varepsilon) \longrightarrow 0$ as $\varepsilon \longrightarrow 0$ and in view of assumption (iv) we can choose $T$ in such a way that the last term of the above estimate are sufficiently small. Thus $H$ is a continuous function on $B C(\Omega)$. To complete the proof we need to verify that $H$ is a compact operator. Let $U$ be a nonempty and bounded subset of $B C(\Omega)$, and assume that $T>0$ and $\varepsilon>0$ are arbitrary constants. Then for $u \in U$ and $x_{1}, x_{2} \in \bar{B}_{T}$, with $\left\|x_{2}-x_{1}\right\| \leq \varepsilon$ we have

$$
\begin{aligned}
&\left|H u\left(x_{2}\right)-H u\left(x_{1}\right)\right| \\
& \leq\left|\int_{\Omega^{\prime}} k\left(x_{2}, y\right) h(y, u(\eta(y))) d y-\int_{\Omega^{\prime}} k\left(x_{1}, y\right) h(y, u(\eta(y))) d y\right| \\
& \leq \int_{\bar{B}_{T}}\left|k\left(x_{2}, y\right)-k\left(x_{1}, y\right)\right||h(y, u(\eta(y)))| d y \\
& \quad+\int_{\Omega^{\prime} \backslash \bar{B}_{T}}\left|k\left(x_{2}, y\right)-k\left(x_{1}, y\right)\right| a(y) b(|u(\eta(y))|) d y \\
& \leq m\left(\bar{B}_{T}\right) U_{r}^{T} \omega_{r}^{T}(k, \varepsilon)+b(r) \int_{\Omega^{\prime} \backslash \bar{B}_{T}}\left[\left|k\left(x_{2}, y\right)\right|+\left|k\left(x_{1}, y\right)\right|\right] a(y) d y
\end{aligned}
$$


where

$$
\begin{aligned}
& r=\sup \{\|u\|: u \in U\}, \\
& \omega_{r}^{T}(k, \varepsilon)=\sup \left\{\left|k\left(x_{1}, y\right)-k\left(x_{2}, y\right)\right|:\right. \\
& \left.x_{1}, x_{2} \in \bar{B}_{T} \subset \Omega,\left\|x_{2}-x_{1}\right\| \leq \varepsilon, y \in \bar{B}_{T} \subset \Omega^{\prime}\right\}, \\
& U_{r}^{T}=\sup \left\{|h(y, u)|: y \in \bar{B}_{T}, u \in[-r, r]\right\} \text {. }
\end{aligned}
$$

Since $u$ was an arbitrary element of $U$ in (3.10), we obtain

$$
\omega^{T}(H(U), \varepsilon) \leq m\left(\bar{B}_{T}\right) U_{r}^{T} \omega_{r}^{T}(k, \varepsilon)+b(r) \int_{\Omega^{\prime} \backslash \bar{B}_{T}}\left[\left|k\left(x_{2}, y\right)\right|+\left|k\left(x_{1}, y\right)\right|\right] a(y) d y
$$

On the other hand, by the uniform continuity of $k$ on the compact set $\bar{B}_{T} \times \bar{B}_{T}$, we have $\omega_{r}^{T}(k, \varepsilon) \longrightarrow 0$ as $\varepsilon \longrightarrow 0$. Therefore, we obtain

$$
\omega_{0}^{T}(H(U)) \leq b(r)\left(\int_{\Omega^{\prime} \backslash \bar{B}_{T}}\left|k\left(x_{2}, y\right)\right| a(y) d y+\int_{\Omega^{\prime} \backslash \bar{B}_{T}}\left|k\left(x_{1}, y\right)\right| a(y) d y\right) .
$$

Now taking $T \longrightarrow \infty$ and by using assumption (iv) we get

$$
\omega_{0}(H(U))=0 .
$$

Moreover, for arbitrary $u, v \in U$ and $x \in \Omega$ we have the following estimate

$$
\begin{aligned}
|H u(x)-H v(x)| & \leq\left|\int_{\Omega^{\prime}} k(x, y)[h(y, u(\eta(y)))-h(y, v(\eta(y)))] d y\right| \\
& \leq \int_{\Omega^{\prime}} k(x, y)[|h(y, u(\eta(y)))|+|h(y, v(\eta(y)))|] d y \\
& \leq \int_{\Omega^{\prime}} k(x, y) a(y)[b(|u(\eta(y))|)+b(|v(\eta(y))|)] d y \\
& \leq 2 b(r) \int_{\Omega^{\prime}} k(x, y) a(y) d y,
\end{aligned}
$$

where $r=\sup \{\|u\|: u \in U\}$. Thus, we obtain

$$
\operatorname{diam} H(U)(x) \leq 2 b(r) \int_{\Omega^{\prime}} k(x, y) a(y) d y .
$$

Taking $\|x\| \longrightarrow \infty$ in the inequality (3.12), then using (iii) we arrive at

$$
\limsup _{\|x\| \longrightarrow \infty} \operatorname{diam} H(U)(x)=0 .
$$

Further, combining (3.11) and (3.13) we get

$$
\limsup _{\|x\| \longrightarrow \infty} \operatorname{diam} H(U)(x)+\omega_{0}(H(U))=0
$$

or, equivalently $\mu(H(U))=0$. Thus, $H$ is a compact operator and the proof is complete.

Finally, we define a compact-integral operator of Volterra-Fredholm type on $B C(\Omega)$. 
Theorem 3.3. Assume that the following conditions are satisfied:

(i) Let $\Omega$ be a nonempty and unbounded subset of the Euclidean space $\mathbb{R}^{n}, \Gamma: \Omega \longrightarrow$ $\mathfrak{M}_{\mathbb{R}^{m}}, \zeta: \Omega^{\prime} \times \bigcup_{x \in \Omega} \Gamma(x) \longrightarrow \Omega$ and $k: \Omega \times \Omega^{\prime} \longrightarrow \mathbb{R}\left(\Omega^{\prime}\right.$ is a subset of $\left.\mathbb{R}^{k}\right)$ are continuous functions such that $\bigcup_{\|x\| \leq T} \Gamma(x)$ is bounded subset of $\mathbb{R}^{m}$ for all $T>0$.

(ii) $g: \Omega^{\prime} \times \bigcup_{x \in \Omega} \Gamma(x) \times \mathbb{R} \longrightarrow \mathbb{R}$ is continuous and there exist a continuous and nondecreasing function $\vartheta: \mathbb{R}_{+} \longrightarrow \mathbb{R}_{+}$such that

$$
\sup \left\{\left|\int_{\Omega^{\prime}} \int_{\Gamma(x)} k(x, y) g(y, z, u(\zeta(y, z))) d z d y\right|: x \in \Omega, u \in \bar{B}_{r}\right\}<\vartheta(r) .
$$

Moreover, for any $r>0$

$$
\lim _{\|x\| \longrightarrow \infty}\left|\int_{\Omega^{\prime}} \int_{\Gamma(x)} k(x, y)[g(y, z, u(\zeta(y, z)))-g(y, z, v(\zeta(y, z)))] d z d y\right|=0,
$$

uniformly respect to $u, v \in \bar{B}_{r}$ and the function

$$
y \longrightarrow \int_{\Gamma(x)} k(x, y) g(y, z, u(\zeta(y, z))) d z
$$

is integrable over $\Omega^{\prime}$ for any fixed $x \in \Omega$,

(iii) The following equality holds:

$$
\begin{aligned}
\lim _{T \rightarrow \infty}\left\{\operatorname { s u p } \left\{\left|\int_{\Omega^{\prime} \backslash \bar{B}_{T}} \int_{\Gamma(x)} k(x, y) g(y, z, u(\zeta(y, z))) d z d y\right|:\right.\right. & \\
x \in \Omega, u \in B C(\Omega)\}\} & =0 .
\end{aligned}
$$

Then $Q: B C(\Omega) \longrightarrow B C(\Omega)$ defined by

$$
Q u(x)=\int_{\Omega^{\prime}} \int_{\Gamma(x)} k(x, y) g(y, z, u(\zeta(y, z))) d z d y
$$

is a compact and continuous operator and $\|Q u\|_{u} \leq \vartheta\left(\|u\|_{u}\right)$.

Proof. Obviously, $Q u(x)$ for any $u \in B C(\Omega)$ is well defined and continuous on $\Omega$, because the function $y \longrightarrow \int_{\Gamma(x)} k(x, y) g(y, z, u(\zeta(y, z))) d z$ is integrable over $\Omega^{\prime}$ for any fixed $x \in \Omega$ and by (3.14), $Q$ is a self operator on $B C(\Omega)$. Now we show that $Q$ is continuous. To do this, take $u \in B C(\Omega)$ and $\varepsilon>0$ arbitrarily. Moreover take $v \in B C(\Omega)$ with $\|u-v\|_{u}<\varepsilon$. Then, considering condition (ii) there exists $T>0$ such that for $\|x\|>T$ we have

$$
\begin{aligned}
& |Q u(x)-Q v(x)| \leq \\
& \qquad\left|\int_{\Omega^{\prime}} \int_{\Gamma(x)} k(x, y)[g(y, z, u(\zeta(y, z)))-g(y, z, v(\zeta(y, z)))] d z d y\right| \leq \varepsilon .
\end{aligned}
$$


Also, if $x \in \bar{B}_{T}$, then we have

$$
\begin{aligned}
|Q u(x)-Q v(x)| & \\
\leq & \left|\int_{\bar{B}_{T}} \int_{\Gamma(x)} k(x, y)[g(y, z, u(\zeta(y, z)))-g(y, z, v(\zeta(y, z)))] d z d y\right| \\
& +\left|\int_{\Omega^{\prime} \backslash \bar{B}_{T}} \int_{\Gamma(x)} k(x, y)[g(y, z, u(\zeta(y, z)))-g(y, z, v(\zeta(y, z)))] d z d y\right| \\
\leq & m\left(\bar{B}_{T}\right) K_{T} \theta_{T}(\varepsilon)+\int_{\Omega^{\prime} \backslash \bar{B}_{T}} \int_{\Gamma(x)} k(x, y)[|g(y, z, u(\zeta(y, z)))| \\
& +|g(y, z, v(\zeta(y, z)))|] d z d y
\end{aligned}
$$

where

$$
\begin{aligned}
& K_{T}=\sup \left\{k(x, y): x \in \bar{B}_{T} \subset \Omega, y \in \bar{B}_{T} \subset \Omega^{\prime}\right\} \\
& \theta_{T}(\varepsilon)=\sup \{|g(y, z, u)-g(y, z, v)|: \\
& \left.y \in \bar{B}_{T} \subset \Omega^{\prime}, z \in \overline{\bigcup_{\|x\| \leq T} \Gamma(x)} u, v \in[-b, b],\|u-v\|_{u} \leq \varepsilon\right\},
\end{aligned}
$$

with $b=\|u\|_{u}+\varepsilon$. By using the continuity of $g$ on the compact set $\bar{B}_{T} \times \overline{\bigcup_{\|x\| \leq T} \Gamma(x)} \times[-b, b]$, we have $\theta_{T}(\varepsilon) \longrightarrow 0$ as $\varepsilon \longrightarrow 0$. On the other hand, in view of assumption (iii) we can choose $T$ in such a way that the last term of the estimate (3.17) are sufficiently small. Thus, $Q$ is a continuous function on $B C(\Omega)$. To complete the proof we need to verify that $Q$ is a compact operator. Let $U$ be a nonempty and bounded subset of $B C(\Omega)$, and assume that $T>0$ and $\varepsilon>0$ are arbitrary constants. Similar to the proof of Theorems 3.1 and 3.2, we deduce

$$
\begin{aligned}
\omega^{T}(Q(U), \varepsilon) \leq & m\left(\bar{B}_{T}\right) \omega^{T}(\Gamma, \varepsilon) K_{T} U_{r}^{T}+m\left(\bar{B}_{T}\right) U_{r}^{T} \omega_{r}^{T}(k, \varepsilon) \\
& +\int_{\Omega^{\prime} \backslash \bar{B}_{T}} \int_{\Gamma\left(x_{2}\right)}\left|k\left(x_{2}, y\right) g(y, z, u(\zeta(y, z)))\right| d z d y \\
& +\int_{\Omega^{\prime} \backslash \bar{B}_{T}} \int_{\Gamma\left(x_{1}\right)}\left|k\left(x_{1}, y\right) g(y, z, u(\zeta(y, z)))\right| d z d y
\end{aligned}
$$

where

$$
\begin{aligned}
& \omega_{r}^{T}(k, \varepsilon)=\sup \left\{\left|k\left(x_{1}, y\right)-k\left(x_{2}, y\right)\right|:\right. \\
& \left.\qquad x_{1}, x_{2} \in \bar{B}_{T} \subset \Omega, y \in \bar{B}_{T} \subset \Omega^{\prime},\left\|x_{1}-x_{2}\right\| \leq \varepsilon\right\}, \\
& \omega^{T}(\Gamma, \varepsilon)=\sup \left\{m(\Gamma(x) \triangle \Gamma(y)): x, y \in \bar{B}_{T}, \| x-y \mid \leq \varepsilon\right\}, \\
& U_{r}^{T}=\sup \left\{|g(y, z, u)|: y \in \bar{B}_{T} \subset \Omega^{\prime}, z \in \bigcup_{\|x\| \leq T} \Gamma(x), u \in[-r, r]\right\},
\end{aligned}
$$

with $r=\sup \left\{\|u\|_{u}: u \in U\right\}$. On the other hand, by the uniform continuity of $k$ and $\Gamma$ on the compact sets $\bar{B}_{T} \times \bar{B}_{T}$ and $\bar{B}_{T}$, respectively, we have $\omega_{r}^{T}(k, \varepsilon) \longrightarrow 0$ and $\omega^{T}(\Gamma, \varepsilon) \longrightarrow 0$ as $\varepsilon \longrightarrow 0$. Therefore, we obtain

$\omega_{0}^{T}(Q(U)) \leq 2 \sup \left\{\int_{\Omega^{\prime} \backslash \bar{B}_{T}} \int_{\Gamma(x)}|k(x, y) g(y, z, u(\zeta(y, z)))| d z d y: x \in \Omega, u \in U\right\}$. 
Now taking $T \longrightarrow \infty$ and by using assumption(iii) we get

$$
\omega_{0}(Q(U))=0
$$

Moreover, we obtain

$$
\begin{array}{r}
\operatorname{diam} Q(U)(x) \leq \\
\sup \left\{\left|\int_{\Omega^{\prime}} \int_{\Gamma(x)} k(x, y)[g(y, z, u(\zeta(y, z)))-g(y, z, v(\zeta(y, z)))] d z d y\right|:\right. \\
u, v \in B C(\Omega)\} .
\end{array}
$$

Taking $\|x\| \longrightarrow \infty$ in the inequality (3.19), then using (ii) we arrive at

$$
\limsup _{\|x\| \rightarrow \infty} \operatorname{diam} Q(U)(x)=0 .
$$

Further, combining (3.18) and (3.20) we get

$$
\limsup _{\|x\| \longrightarrow \infty} \operatorname{diam} Q(U)(x)+\omega_{0}(Q(U))=0
$$

or, equivalently $\mu(Q(U))=0$. Thus, $Q$ is a compact operator and the proof is complete.

\section{Application}

Theorem 4.1. Assume that the following conditions are satisfied:

(i) Let $\Omega$ be a nonempty and unbounded subset of the Euclidean space $\mathbb{R}^{n}, f_{1}: \Omega \times$ $\mathbb{R} \longrightarrow \mathbb{R}$ and $f_{2}: \Omega \times \mathbb{R} \times \mathbb{R} \times \mathbb{R} \longrightarrow \mathbb{R}$ are continuous. Moreover there exist $k_{1}, k_{2} \in[0,1]$, and nondecreasing, concave and right continuous functions $\varphi: \mathbb{R}_{+} \longrightarrow \mathbb{R}_{+}$such that $\varphi(t)<t$ for all $t \geq 0$,

$$
\left|f_{1}\left(x, u_{1}\right)-f_{1}\left(x, u_{2}\right)\right| \leq k_{1} \varphi\left(\left|u_{1}-u_{2}\right|\right)
$$

and

$$
\left|f_{2}\left(x, u_{1}, v_{1}, z_{1}\right)-f_{2}\left(x, u_{2}, v_{2}, z_{2}\right)\right| \leq k_{2} \varphi\left(\left|u_{1}-u_{2}\right|\right)+\left|v_{1}-v_{2}\right|+\left|z_{1}-z_{2}\right| .
$$

(ii) $M_{1}:=\sup \left\{\left|f_{1}(x, 0)\right|: x \in \Omega\right\}<\infty$ and $M_{2}:=\sup \left\{\left|f_{2}(x, 0,0,0)\right|\right.$ : $x \in \Omega\}<\infty$.

(iii) $G_{i}: B C(\Omega) \longrightarrow B C(\Omega)(i=1,2,3)$ be a compact and continuous operator and there exists a nondecreasing function $\vartheta_{i}: \mathbb{R}_{+} \longrightarrow \mathbb{R}_{+}$such that

$$
\left\|G_{i}(x)\right\| \leq \vartheta_{i}(\|x\|)
$$


(iv) There exists a positive solution $r_{0}$ such that

$$
k_{1} \varphi(r)+M_{1}+\left(M_{2}+k_{2} \varphi(r)+\vartheta_{1}(r)+\vartheta_{2}(r)\right) \vartheta_{3}(r) \leq r
$$

and

$$
k_{1}+k_{2} \vartheta_{3}(r) \leq 1
$$

Then there exists $u \in B C(\Omega)$ such that

$$
u(x)=f_{1}(x, u(x))+f_{2}\left(x, u(x), G_{1} u(x), G_{2} u(x)\right) G_{3} u(x) .
$$

Proof. Define the operator $F: B C(\Omega) \longrightarrow B C(\Omega)$ by

$$
F u(x)=f_{1}(x, u(x))+f_{2}\left(x, u(x), G_{1} u(x), G_{2} u(x)\right) G_{3} u(x) .
$$

Obviously, $F u(x)$ for any $u \in B C(\Omega)$ is well defined and continuous on $\Omega$. Since $G_{i}$ and $f_{i}$ are continuous, therefore, $F$ is a continuous operator on $B C(\Omega)$. Using conditions (i)-(iii), for arbitrarily fixed $x \in \Omega$, we have

$|F u(x)|$

$$
\begin{aligned}
\leq & \left|f_{1}(x, u(x))-f_{1}(x, 0)\right|+\left|f_{1}(x, 0)\right| \\
& +\left(\left|f_{2}\left(x, u(x), G_{1} u(x), G_{2} u(x)\right)-f_{2}(x, 0,0,0)\right|+\left|f_{2}(x, 0,0,0)\right|\right)\left|G_{3} u(x)\right| \\
\leq & k_{1} \varphi(|u(x)|)+M_{1}+\left(M_{2}+k_{2} \varphi(|u(x)|)+\left|G_{1} u(x)\right|+\left|G_{2} u(x)\right|\right)\left|G_{3} u(x)\right| \\
\leq & k_{1} \varphi(\|u\|)+M_{1}+\left(M_{2}+k_{2} \varphi(\|u\|)+\vartheta_{1}(\|u\|)+\vartheta_{2}(\|u\|)\right) \vartheta_{3}(\|u\|)
\end{aligned}
$$

Thus, we obtain

$$
\|F u\| \leq k_{1} \varphi(\|u\|)+M_{1}+\left(M_{2}+k_{2} \varphi(\|u\|)+\vartheta_{1}(\|u\|)+\vartheta_{2}(\|u\|)\right) \vartheta_{3}(\|u\|),
$$

and $F u \in B C(\Omega)$ for any $u \in B C(\Omega)$. Thus, keeping in mind assumption (iv), we infer that $F$ is a self mapping of the ball $\bar{B}_{r_{0}}$. Now we show that for any nonempty set $U \subset \bar{B}_{r_{0}}$ we have $\omega_{0}(F(U)) \leq \varphi\left(\omega_{0}(U)\right)$.

For this, we fix $T>0$ and $\varepsilon>0$ arbitrarily. In similar argument we have

$$
\begin{aligned}
\omega^{T}(F(U), \varepsilon) \leq & \omega_{r_{0}}^{T}\left(f_{1}, \varepsilon\right)+\vartheta_{3}\left(r_{0}\right) \omega_{r_{0}}^{T}\left(f_{2}, \varepsilon\right)+\left(k_{1}+k_{2} \vartheta_{3}\left(r_{0}\right)\right) \varphi\left(\omega^{T}(U, \varepsilon)\right) \\
& +\vartheta_{3}\left(r_{0}\right)\left(\omega^{T}\left(G_{1}(U), \varepsilon\right)+\omega^{T}\left(G_{2}(U), \varepsilon\right)\right) \\
& +\left(\varphi\left(r_{0}\right)+\vartheta_{1}\left(r_{0}\right)+\vartheta_{2}\left(r_{0}\right)+M_{2}\right) \omega^{T}\left(G_{3}(U), \varepsilon\right)
\end{aligned}
$$

where

$$
\begin{gathered}
\omega_{r_{0}}^{T}\left(f_{1}, \varepsilon\right)=\sup \left\{\left|f_{1}(x, u)-f_{1}(y, u)\right|: x, y \in \bar{B}_{T}, u \in\left[-r_{0}, r_{0}\right]\right\} \\
\omega_{r_{0}}^{T}\left(f_{2}, \varepsilon\right)=\sup \left\{\left|f_{2}(x, u, v, z)-f_{2}(y, u, v, z)\right|: x, y \in \bar{B}_{T}, u \in\left[-r_{0}, r_{0}\right],\right. \\
\left.v \in\left[-\vartheta_{1}\left(r_{0}\right), \vartheta_{1}\left(r_{0}\right)\right], z \in\left[-\vartheta_{2}\left(r_{0}\right), \vartheta_{2}\left(r_{0}\right)\right]\right\} .
\end{gathered}
$$


By using the continuity of $f_{1}$ and $f_{2}$ on the compact set $\bar{B}_{T} \times\left[-r_{0}, r_{0}\right]$ and $\bar{B}_{T} \times\left[-r_{0}, r_{0}\right] \times\left[-\vartheta_{1}\left(r_{0}\right), \vartheta_{1}\left(r_{0}\right)\right] \times\left[-\vartheta_{2}\left(r_{0}\right), \vartheta_{2}\left(r_{0}\right)\right]$, respectively, we have $\omega_{r_{0}}^{T}\left(f_{i}, \varepsilon\right) \longrightarrow 0$ as $\varepsilon \longrightarrow 0$, and

$$
\begin{aligned}
\omega_{0}^{T}(F(U)) \leq & \left(k_{1}+k_{2} \vartheta_{3}\left(r_{0}\right)\right) \varphi\left(\omega_{0}^{T}(U)\right)+\vartheta_{3}\left(r_{0}\right)\left(\omega_{0}^{T}\left(G_{1}(U)\right)+\omega_{0}^{T}\left(G_{2}(U)\right)\right) \\
& +\left(\varphi\left(r_{0}\right)+\vartheta_{1}\left(r_{0}\right)+\vartheta_{2}\left(r_{0}\right)+M_{2}\right) \omega_{0}^{T}\left(G_{3}(U)\right) .
\end{aligned}
$$

Also, since $G_{i}$ are compact operators for all $1 \leq i \leq 3$, so we have $\omega_{0}^{T}\left(G_{i}(U)\right) \longrightarrow$ 0 as $T \longrightarrow \infty$, and

$$
\omega_{0}(F(U)) \leq\left(k_{1}+k_{2} \vartheta_{3}\left(r_{0}\right)\right) \varphi\left(\omega_{0}(U)\right) .
$$

Moreover, for arbitrary $u, v \in U$ and $x \in \Omega$, we have the following estimate

$$
\begin{aligned}
|F u(x)-F v(x)| \leq & \left(k_{1}+k_{2} \vartheta_{3}\left(r_{0}\right)\right) \varphi(\operatorname{diam} U(x)) \\
& +\vartheta_{3}\left(r_{0}\right)\left(\operatorname{diam} G_{1}(U)(x)+\operatorname{diam}_{2}(U)(x)\right) \\
& +\left(\varphi\left(r_{0}\right)+\vartheta_{1}\left(r_{0}\right)+\vartheta_{2}\left(r_{0}\right)+M_{2}\right) \operatorname{diam} G_{3}(U)(x) .
\end{aligned}
$$

Thus, we obtain

$$
\begin{aligned}
\operatorname{diam} F(U)(x) \leq & \left(k_{1}+k_{2} \vartheta_{3}\left(r_{0}\right)\right) \varphi(\operatorname{diam} U(x)) \\
& +\vartheta_{3}\left(r_{0}\right)\left(\operatorname{diam} G_{1}(U)(x)+\operatorname{diam} G_{2}(U)(x)\right) \\
& +\left(\varphi\left(r_{0}\right)+\vartheta_{1}\left(r_{0}\right)+\vartheta_{2}\left(r_{0}\right)+M_{2}\right) \operatorname{diam} G_{3}(U)(x) .
\end{aligned}
$$

Taking $\|x\| \longrightarrow \infty$ in the inequality (4.6), and using $\lim \sup \operatorname{diam} G_{i}(X) \longrightarrow 0$ $(1 \leq i \leq 3)$, we have

$$
\limsup _{\|x\| \longrightarrow \infty} \operatorname{diam} F(U)(x) \leq\left(k_{1}+k_{2} \vartheta_{3}\left(r_{0}\right)\right) \varphi\left(\limsup _{\|x\| \longrightarrow \infty} \operatorname{diam} U(x)\right) .
$$

Further, combining (4.5) and (4.7) we get

$$
\begin{aligned}
\limsup _{\|x\| \rightarrow \infty} \operatorname{diam} F(U)(x)+ & \omega_{0}(F(U)) \leq \\
\left(k_{1}+k_{2} \vartheta_{3}\left(r_{0}\right)\right) & \left(\varphi\left(\limsup _{\|x\| \rightarrow \infty} \operatorname{diam} U(x)\right)+\varphi\left(\omega_{0}(U)\right)\right) .
\end{aligned}
$$

Since $\varphi$ is a concave function, (4.8) and (iv) imply that

$$
\limsup _{\|x\| \longrightarrow \infty} \operatorname{diam} F(U)(x)+\omega_{0}(F(U)) \leq 2 \varphi\left(\frac{1}{2} \omega_{0}(U)+\frac{1}{2} \limsup _{\|x\| \rightarrow \infty} \operatorname{diam} U(x)\right) .
$$

Consequently, by considering $\mu$ defined by the formula

$$
\mu(U)=\frac{1}{2} \omega_{0}(U)+\frac{1}{2} \limsup _{\|x\| \longrightarrow \infty} \operatorname{diam} U(x)
$$

we get

$$
\mu(F(U)) \leq \varphi(\mu(U)) .
$$

Thus from Theorem 2.1 we conclude that the operator $F$ has a fixed point $u$ in $\bar{B}_{r_{0}}$, and thus Eq. (4.4) has at least a solution in $B C(\Omega)$. 
As a consequence of Theorems 3.1, 3.2 and 4.1, we obtain the following results.

Corollary 4.2. Let all hypotheses of Theorems 3.1 and 3.2 are satisfied and $f_{2}: \Omega \times \mathbb{R} \times \mathbb{R} \times \mathbb{R} \longrightarrow \mathbb{R}$ be a continuous function. Moreover there exists a nondecreasing, concave and right continuous function $\varphi: \mathbb{R}_{+} \longrightarrow \mathbb{R}_{+}$such that $\varphi(t)<t$ for all $t \geq 0$,

$$
\left|f_{2}\left(x, u_{1}, v_{1}, z_{1}\right)-f_{2}\left(x, u_{2}, v_{2}, z_{2}\right)\right| \leq \varphi\left(\left|u_{1}-u_{2}\right|\right)+\left|v_{1}-v_{2}\right|+\left|z_{1}-z_{2}\right|
$$

and

$$
M:=\sup \left\{\left|f_{2}(x, 0,0,0)\right|: x \in \Omega\right\}<\infty .
$$

Also, there exists a positive solution $r_{0}$ of the inequality

$$
M+\varphi(r)+\vartheta(r)+D b(r) \leq r .
$$

Then Eq. (1.6) has at least a solution in the space $B C(\Omega)$.

Proof. Consider $G_{1}, G_{2}: B C(\Omega) \longrightarrow B C(\Omega)$ by the formulas

$$
\begin{aligned}
& G_{1} u(x)=\int_{\Gamma(x)} g(x, y, u(\xi(y))) d y \\
& G_{2} u(x)=\int_{\Omega^{\prime}} k(x, y) h(y, u(\eta(y))) d y .
\end{aligned}
$$

Then by applying Theorems 4.1, 3.1 and 3.2, we see that Eq. (1.6) has at least a solution in the space $B C(\Omega)$.

Corollary 4.3. Let all hypotheses of Theorem 3.3 are satisfied and $f_{i}: \Omega \times \mathbb{R} \longrightarrow \mathbb{R}$ $(i=1,2)$ be a continuous function. Moreover there exists a nondecreasing, concave and right continuous function $\varphi: \mathbb{R}_{+} \longrightarrow \mathbb{R}_{+}$and $k_{1}, k_{2} \in[0,1]$ such that $\varphi(t)<t$ for all $t \geq 0$,

$$
\left|f_{i}\left(x, u_{1}\right)-f_{i}\left(x, u_{2}\right)\right| \leq k_{i} \varphi\left(\left|u_{1}-u_{2}\right|\right) .
$$

There exists a positive solution $r_{0}$ such that

$$
M_{1}+k_{1} \varphi(r)+\left(M_{2}+k_{2} \varphi(r)\right) \vartheta(r) \leq r,
$$

and

$$
k_{1}+k_{2} \vartheta(r) \leq 1
$$

Also

$$
M_{i}:=\sup \left\{\left|f_{i}(x, 0)\right|: x \in \Omega\right\}<\infty .
$$

Then the Eq. (1.7) has at least a solution in the space $B C(\Omega)$.

Proof. The proof is similar to the proof of the previous Corollaries.

Now, we give the following examples which also show that our results can be applied but the previous results $[1,3,4,6,7,11,14,17,18,20]$ are inapplicable. 
Example 4.1. Consider the following functional integral equation

$$
\begin{aligned}
x(t)=e^{-t^{2}} \ln (1+ & |x(t)|)+\int_{-t^{2}}^{|t|} s e^{-s^{2}} \sqrt{x\left(s^{2}\right)} d s+ \\
& \tanh \left(\int_{\mathbb{R}_{+}} \int_{\mathbb{R}} \frac{u \cos s t}{1+t^{2}} e^{-\left(s+u^{2}\right)} \sqrt[3]{x(u s) \arctan u s} d u d s\right) .
\end{aligned}
$$

Now, we study the solvability of Eq. (4.10) on the space $B C(\mathbb{R})$. Observe that this integral equation is a special case of Eq. (1.6) with

$$
\begin{aligned}
& \xi(s)=s^{2}, \quad \eta(s, u)=u s, \quad \Omega^{\prime}=\mathbb{R}_{+} \times \mathbb{R}, \quad \Gamma(t)=\left[-t^{2},|t|\right], \\
& f_{2}(t, x, y, z)=e^{-t^{2}} \ln (1+|x|)+y+\tanh z, \\
& g(t, s, x)=s e^{-s^{2}} \sqrt{x}, \quad k(t, s, u)=\frac{u \cos s t}{1+t^{2}} e^{-\left(s+u^{2}\right)}, \quad h(s, u, x)=\sqrt[3]{x \arctan u s} .
\end{aligned}
$$

Taking $\varphi(t)=\ln (1+|t|), \vartheta(t)=\sqrt{t}, a(s, u)=\sqrt[3]{\arctan u s}$ and $b(x)=\sqrt[3]{x}$. Now, by some simple calculations, we show that all the assumptions of Corollary 4.2 hold. Clearly Some assumptions are valid due to the selected functions. We are just going to check critical assumptions. For this purpose, we obtain $M=1$,

$$
\sup \left\{\left|\int_{-t^{2}}^{|t|} s e^{-s^{2}} \sqrt{x\left(s^{2}\right)} d s\right|: t \in \mathbb{R}, x \in B C\left(\mathbb{R}_{+}\right),\|x\| \leq r\right\} \leq \sqrt{r}
$$

Moreover, for any $r>0$ we have

$$
\lim _{|t| \rightarrow \infty} \int_{-t^{2}}^{|t|} s e^{-s^{2}}\left[\sqrt{x\left(s^{2}\right)}-\sqrt{y\left(s^{2}\right)}\right] d s=0
$$

uniformly with respect to $x, y \in \bar{B}_{r}$. Thus, condition (ii) of Theorem 3.1 is true. Further, we have

$$
\int_{\mathbb{R}_{+}} \int_{\mathbb{R}}\left|\frac{u \cos s t \sqrt[3]{\arctan u s}}{1+t^{2}} e^{-\left(s+u^{2}\right)}\right| d u d s \leq \frac{\sqrt[3]{\frac{\pi}{2}}}{1+t^{2}} \leq \sqrt[3]{\frac{\pi}{2}}
$$

and we obtain $D=\sqrt[3]{\frac{\pi}{2}}$. On the other hand,

$$
\lim _{|t| \rightarrow \infty} \int_{\mathbb{R}_{+}} \int_{\mathbb{R}}\left|\frac{u \cos s t \sqrt[3]{\arctan u s}}{1+t^{2}} e^{-\left(s+u^{2}\right)}\right| d u d s=0 .
$$

Consequently, assumption (iii) of Theorem 3.2 is satisfied. We now show that assumption (iv) of Theorem 3.2 holds. For arbitrarily fixed $T>0$, there exists $T^{\prime}>0$ such that $\Omega^{\prime} \backslash \bar{B}_{T} \subset \Omega^{\prime} \backslash\left(\left[0, T^{\prime}\right] \times\left[-T^{\prime}, T^{\prime}\right]\right)$ and therefore, we get:

$$
\begin{aligned}
\int_{\Omega^{\prime} \backslash\left(\left[0, T^{\prime}\right] \times\left[-T^{\prime}, T^{\prime}\right]\right)}\left|\frac{u \cos s t \sqrt[3]{\arctan u s}}{1+t^{2}} e^{-\left(s+u^{2}\right)}\right| d u d s & \int_{T^{\prime}}^{\infty} \int_{\mathbb{R}}\left|\frac{u \cos s t \sqrt[3]{\arctan u s}}{1+t^{2}} e^{-\left(s+u^{2}\right)}\right| d u d s \\
& +2 \int_{0}^{T^{\prime}} \int_{T^{\prime}}^{\infty}\left|\frac{u \cos s t \sqrt[3]{\arctan u s}}{1+t^{2}} e^{-\left(s+u^{2}\right)}\right| d u d s \\
\leq & {\left[e^{-T^{\prime}}+\left(1-e^{-T^{\prime}}\right) e^{-\left(T^{\prime}\right)^{2}}\right] \sqrt[3]{\frac{\pi}{2}} }
\end{aligned}
$$


for all $t \in \mathbb{R}$. Thus, we infer that assumption (iv) of Theorem 3.2 holds. Finally, let us notice that the inequality from Corollary 4.2 , having the form

$$
M+\varphi(r)+\vartheta(r)+D b(r) \leq 1+\ln (1+r)+\sqrt{r}+\left[\sqrt[3]{\frac{\pi}{2}}\right] \sqrt[3]{r} \leq r .
$$

It is easy to see that each number $r \geq 9$ (this estimate can be improved) satisfies the above inequality. Thus, as the number $r_{0}$ we can take $r_{0}=9$. Consequently, all the assumptions in Corollary 4.2 are provided. Hence Eq. (4.10) has at least a solution belongs to the space $B C(\mathbb{R})$.

Example 4.2. Consider the following functional integral equation

$$
\begin{aligned}
& x(t, s)=\frac{s t^{2}|x(t, s)|}{\left(s^{2} t^{4}+1\right)(|x(t, s)|+1)}+ \\
& \ln (\sqrt{|x(t, s)|+1}) \int_{\mathbb{R}} \int_{0}^{|t s|} \frac{4 u^{3} v e^{-v^{2}}}{\left(s^{8} t^{8}+1\right)(2+\sin x(v, u))} d u d v .
\end{aligned}
$$

Eq. (4.11) is a special case of Eq. (1.7) with

$$
\begin{aligned}
& \zeta(t, s)=(t, s), \quad \Gamma(t, s)=[0,|t s|], \quad \Omega^{\prime}=\mathbb{R}, \\
& f_{1}(t, s, x)=\frac{s t^{2}|x|}{\left(s^{2} t^{4}+1\right)(|x|+1)}, \quad f_{2}(t, s, x)=\ln (\sqrt{|x|+1}), \\
& k(t, s, v)=\frac{v e^{-v^{2}}}{s^{8} t^{8}+1}, \quad g(v, u, x)=\frac{4 u^{3}}{2+\sin x} .
\end{aligned}
$$

Taking $k_{1}, k_{2}=\frac{1}{2}, \varphi(t)=\max \left\{\ln (t+1), \frac{t}{t+1}\right\}$ and $\vartheta(t)=1$. We investigate that all the assumptions of Corollary 4.3 hold. To do this, we obtain $M_{i}=0$,

$$
\left|\int_{\mathbb{R}} \int_{0}^{|t s|} \frac{4 u^{3} v e^{-v^{2}}}{\left(s^{8} t^{8}+1\right)(2+\sin x(s, u))} d u d v\right| \leq \frac{t^{4} s^{4}}{s^{8} t^{8}+1}
$$

and we deduce

$$
\begin{gathered}
\sup \left\{\left|\int_{\mathbb{R}} \int_{0}^{|t s|} \frac{4 u^{3} v e^{-v^{2}}}{\left(s^{8} t^{8}+1\right)(2+\sin x(s, u))} d u d v\right|: t, s \in \mathbb{R}, x \in B C\left(\mathbb{R}^{2}\right),\|x\| \leq r\right\}<1, \\
\lim _{\|(t, s)\| \longrightarrow \infty}\left|\int_{\mathbb{R}} \int_{0}^{|t s|} \frac{4 u^{3} v e^{-v^{2}}}{\left(s^{8} t^{8}+1\right)(2+\sin x(s, u))}-\frac{4 u^{3} v e^{-v^{2}}}{\left(s^{8} t^{8}+1\right)(2+\sin y(s, u))} d u d v\right|=0 .
\end{gathered}
$$

Consequently, assumption (ii) of Theorem 3.3 is satisfied. On the other hand, for arbitrarily fixed $T>0$ we get:

$$
\begin{gathered}
\left|\int_{\mathbb{R} \backslash \bar{B}_{T}} \int_{0}^{|t s|} \frac{4 u^{3} v e^{-v^{2}}}{\left(s^{8} t^{8}+1\right)(2+\sin x(v, u))} d u d v\right| \\
\leq 2\left|\int_{T}^{\infty} \int_{0}^{|t s|} \frac{4 u^{3} v e^{-v^{2}}}{\left(s^{8} t^{8}+1\right)(2+\sin x(v, u))} d u d v\right| \\
\leq e^{-T^{2}}
\end{gathered}
$$


From the above estimates we infer that assumption (iii) of Theorem 3.3 holds. It is easy to see that each number $r \geq 1$ (this estimate can be improved) satisfies in the following inequalities, i.e.,

$$
M_{1}+k_{1} \varphi(r)+\left(M_{2}+k_{2} \varphi(r)\right) \vartheta(r) \leq \max \left\{\ln (r+1), \frac{r}{r+1}\right\} \leq r,
$$

and

$$
k_{1}+k_{2} \vartheta(r) \leq 1
$$

Thus, as the number $r_{0}$ we can take $r_{0}=1$. Consequently, all assumptions in Corollary 4.3 are provided. Hence Eq. (4.11) has at least a solution belonging to the space $B C(\mathbb{R} \times \mathbb{R})$.

\section{References}

[1] R. P. Agarwal, J. Banaś, B. C. Dhage, and S. D. Sarkate, Attractivity results for a nonlinear functional integral equation, Georgian Mathematical Journal, vol. 18, no. 1, pp. 119, 2011.

[2] A. Aghajani, J. Banaś, Y. Jalilian, Existence of solutions for a class of nonlinear Volterra singular integral equations, Comput. Math. Appl., (2011), 1215-1227.

[3] A. Aghajani, J. Banaś, N. Sabzali, Some generalizations of Darbo fixed point theorem and applications, Bull. Belg. Math. Soc. Simon Stevin, 2013; 20, 2: 345-358.

[4] A. Aghajani, Y. Jalilian, Existence and global attractivity of solutions of a nonlinear functional integral equation, Commun. Nonlin. Sci. Numer. Simul. 15 (2010) 3306-3312.

[5] A. Aghajani, Y. Jalilian, K. Sadarangani, Existence of solutions for mixed Volterra-Fredholm integral equations, Electronic Journal of Differential Equations, no. 137, pp. 1-12, 2012.

[6] A. Aghajani, M. Mursaleen, A. Shole Haghighi, Fixed point theorems for Meir-Keeler condensing operators via measure of noncompactness, Acta Mathematica Scientia (Accepted).

[7] A. Aghajani, A. Shole Haghighi, Existence of solutions for a class of functional integral equations of Volterra type in two variables via measure of noncompactness, IJST, 38A1 (2014), 1-8.

[8] R. Arab, The Existence of Fixed Points via the Measure of Noncompactness and its Application to Functional-Integral Equations, Mediterr. J. Math. DOI 10.1007/s00009-014-0506-y.

[9] J. Banaś, Measures of noncompactness in the space of continuous tempered functions, Demonstratio Math., 14 (1981), 127-133. 
[10] J. Banaś, J. Caballero, J. Rocha, K. Sadarangani, Monotonic solutions of a class of quadratic integral equations of Volterra type, Comput. Math. Appl. 49(5-6) (2005), 943-952.

[11] J. Banaś, B. C. Dhage, Global asymptotic stability of solutions of a functional integral equation, Nonlin. Anal. 69 (2008) 1945-1952.

[12] J. Banaś, K. Goebel, Measures of noncompactness in Banach spaces, Lect. Notes Pure Appl. Math., vol. 60, Dekker, New York, 1980.

[13] J. Banaś, D. O'Regan, On existence and local attractivity of solutions of a quadratic Volterra integral equation of fractional order, J. Math. Anal. Appl. 345 (2008) 573-582.

[14] J. Banaś, R. Rzepka, An application of a measure of noncompactness in the study of asymptotic stability, Appl. Math. Lett. 16 (2003) 1-6.

[15] J. Banaś, K. Sadarangani, Solvability of Volterra-Stieltjes operator-integral equations and their applications, Comput. Math. Appl. 41 (2001) 1535-1544.

[16] J. Banaś, D. O’Regan , K. Sadarangani, On solutions of a quadratic Hammerstein integral equation on an unbounded interval, Dynamic Systems and Applications 18 (2009) 251-264.

[17] B. C. Dhage, Attractivity and positivity results for nonlinear functional integral equations via measure of noncompactness, Differ. Equ. Appl. 2 (2010), 299-318.

[18] E. M. El-Abd, On the existence of solutions for nonlinear functional integral equation, Filomat 24 (2010), no. 4, 17-23.

[19] L. Liu, F. Guo, C. Wu, Y. Wu, Existence theorems of global solutions for nonlinear volterra type integral equations in Banach spaces. J. Math. Anal. Appl. 309 (2005) 638-649.

[20] Z. Xia, Global Asymptotic Stability for Nonlinear Functional Integral Equation of Mixed Type, Hindawi Publishing Corporation, Journal of Applied Mathematics, Volume 2013, Article ID 193602, 11 pages, http:/ /dx.doi.org/10.1155/2013/193602.

Department of Mathematics, Mashhad Branch, Islamic Azad University,

Mashhad, Iran.

emails : rezaallahyari@mshdiau.ac.ir, ali.sholehaghighi@gmail.com

Department of Mathematics, Sari Branch,

Islamic Azad University, Sari, Iran.

email : mathreza.arab@iausari.ac.ir 